Open Access | Research Article

\title{
Primary biliary cholangitis: From diagnosis to treatment
}

\author{
*Corresponding Author(s): Maria Guarino, \\ Department of Clinical Medicine and Surgery, \\ Gastroenterology Unit, University of Naples, Italy \\ Email: maria.guarino86@gmail.com
}

Received: Dec 04, 2017

Accepted: Jan 30, 2018

Published Online: Feb 15, 2018

Journal: Annals of Gastroenterology and the Digestive System Publisher: MedDocs Publishers LLC

Online edition: http://meddocsonline.org/

Copyright: (C) Guarino M (2018). This Article is distributed under the terms of Creative Commons Attribution 4.0 International License

Keywords: Primary biliary cholangitis; PBC management; UDCA; AMA; Obeticholic acid.

\section{Introduction}

Primary biliary cholangitis (PBC; until recently known as primary biliary cirrhosis) [1] is a chronic, progressive, auto immune liver disease characterized by intrahepatic small and mediumsized bile ducts destruction which leads to cholestasis, complications and symptoms related to cholestasis, cirrhosis, and portal hypertension. It is a non-suppurative, destructive cholangitis that attacks the adjacent hepatic parenchyma, gradually advancing to fibrosis and cirrhosis. PBC is often considered a model auto immune disease because of its hallmark serologic signature, the Antimitochondrial Antibody (AMA) and specific bile duct pathology. The PBC is characterized by female pre-

\begin{abstract}
Primary biliary cholangitis (PBC), previously known as primary biliary cirrhosis and which has been designated an orphan condition, is a chronic autoimmune disease resulting in the destruction of the small bile ducts in the liver. Like other complex disorders, PBC is heterogeneous in its pre-sensation, symptomatology, disease progression and response to therapy. Without effective treatment, disease progression frequently leads to liver failure and death. Until May 2016, when Obeticholic acid (OCA) has been licensed as treatment options for patients with PBC, the only FDA-approved treatment for PBC was Ursodeoxycholic acid (UDCA), an oral hydrophilic bile acid, which can slow progression of liver damage due to PBC. However, 1 out of 3 patients taking UDCA has an inadequate biochemical response, leading to increased risk of disease progression, liver transplantation, and mortality. Given this unmet clinical need, new therapies are in development for the treatment of PBC. There are molecules targeting the immune-mediated response and bile-acid therapies targeting the bile acid biosynthetic and feedback processes that drive the biliary epithelial cells injury. In addition, antifibrotic therapies are under study to tackle the downstream damage following biliary injury. In conclusion, $\mathrm{PBC}$ is a condition with a heterogeneous, poorly characterized phenotype for which limited therapeutic options are currently available.
\end{abstract}

dominance (with an 8:1 female-to-male ratio), characteristic antibodies (AMA), elevated immunoglobulin $M$, symptoms like pruritus and fatigue, a cholestatic liver enzyme profile (with elevated alkaline phosphatase [ALP], gamma glutamyl transferase [GGT] as well as serum bilirubin levels), and a prominent feature of extra hepatic immune-mediated disease associations. The median age of diagnosis is approximately 50 years $[2,3]$. The incidence of this disease is increasing. One of the reasons for this increase is the fact that physicians are becoming better at diagnosing PBC earlier. There is a lot of variability in how common this disease is. Most estimates are between 12 and 20 per 100,000 individuals, with the highest estimates exceeding 60 per 100,000 individuals [4-6]. Additionally, even if PBC is 
therefore more commonly observed in Australian Caucasians, it occurs in all ethnic groups with differences in the incidence among countries or ethnicities (incidence rate of 8.9 per million person-years in China [7] and 3.3-58 per million person-years in Western Countries [4]. Even at early stages, patients with PBC can have disabling symptoms of fatigue and pruritus. As the disease progresses and cirrhosis ensues, these patients develop the same complications as other patients with cirrhosis (i.e., ascites, encephalopathy, variceal bleeding, and liver cancer). There is also a myriad of associated conditions that cause additional morbidity, such as osteoporosis, Siccasyndrome, thyroid disorders, and arthritis. For all these reasons, the correct management of patients with PBC has the goal of improving natural history and survival. This can be achieved through:

A) Early diagnosis of PBC in order to start an optimal treatment

b) Prevention of the disease progression with all its complications

\section{PBC Diagnosis}

The diagnostic process should follow a step-by-step approach, beginning with the anamnesis and physical examination, followed by laboratory tests and non-invasive assessement, and finishing with invasive evaluation (as liver biopsy) when needed. The differential diagnosis includes a cholestatic drug reaction, biliary obstruction, sarcoidosis, autoimmune hepatitis and primary sclerosing cholangitis.

\section{Anamnesis:}

- Familiar history of cholestatic liver diseases, or autoimmune diseases, or cirrhosis and liver cancer

\section{- Lifestyle: cigarettes smoking (smoke increase the risk)}

- Careful evaluation of all the patient comorbidities, specially the autoimmune ones- Symptomatology: even if usually the $\mathrm{PBC}$ is asymptomatic, it is necessary to investigate the presence of one or more of the following symptoms - pruritus (usually intermittent during the day and most trouble some in the evening and at night; sometimes severe and intractable), fatigue, anorexia, upper quadrant abdominal pain or discomfort, altered sleep pattern, bone pain with or without spontaneous fractures (for vitamin D deficiency and osteoporosis). Sometimes PBC is initially diagnosed during pregnancy prompted by the symptom of pruritus that fails to disappear in the post partum period, unlike intrahepatic cholestasis of pregnancy.

\section{Physical findings:}

Skin/general aspect: excoriations; skin hyper pigmentation (caused by deposition of melanin); xanthelasma; xerophthalmia; xerostomia; scleral icterus; jaundice; signs of cirrhosis as palmar erythema, spider nevi, muscle wasting. Very rarely, patients have kayser-Fleischer rings because of copper retention.

Abdomen: hepatomegaly; splenomegaly; signs of cirrhosis and portal hypertension as abdominal wall vascular collaterals (caput medusa), as cites

\section{Biochemical features:}

the routine laboratory abnormalities in PBC are typical of chronic cholestatic syndromes and chronic liver disease. Serum ALP and GGTare raised and serum aminotransferase can also be elevated. Serum bilirubin levels usually are normal in early stag- es of PBC and increase slowly over the course of the disease. Elevated levels of immunoglobulin and of cholesterol are common in PBC. Liver synthetic function, i.e.: changes in albumin and INR are observed with disease progression. Moreover, it is possible to find an increase in high-density and low-density lipoprotein cholesterol. Thrombocytopenia, if present, is usually a manifestation of portal hypertension. PBC, like other cholestatic syndromes, leads to copper retention, resulting in raised urinary copper and serum ceruloplasmin levels.

\section{Serologic tests:}

The presence of auto antibodies AMAs is a clinical hall mark and important diagnostic feature of PBC. AMA is found in nearly $95 \%$ of patients with PBC. In approximately $5 \%-10 \%$ of the patients, AMA antibodies are absent or present only in low titer (< 1/40). Non-specific antinuclear antibodies (ANA) are found in at least $30 \%$ of PBC sera. However, ANA directed against nuclear body or envelope proteins such as anti-Sp100 and anti-gp210 show a high specificity for PBC ( $>95 \%)$ and can be used as markers of PBC when AMA are absent. Other autoantibodie that have been reported in patients with $\mathrm{PBC}$ include those with associated autoiimune syndromes.

\section{Liver biopsy and histopathologic features:}

Currently, a diagnosis of PBC can be made with confidence on the basis of two of three following criteria: the presence of biochemical cholestasis (elevation of ALP of liver origin for at least 6 months), presence of AMA (>1:40) and typical histological features of florid bile duct lesions. Aliver biopsy is not essential in patients with ALP elevation and AMA, but may be required for the diagnosis of concurrent features of autoimmune hepatitis and disease stage. PBC is histological characterized by autoimmune-mediated progressive destruction of the intrahepatic small bile ducts, as well as by chronic intrahepatic cholestasis, hepatocellular damage, fibrosis, and sepal formation. Histological lesions are classically divided into 4 stages. Stage $I$ is characterized by portal inflammation with or without florid bile duct lesions, and without fibrosis. Stage II is characterized by the increase of periportalfibrosis extending into the hepatic parenchyma referred to as interface hepatitis. Stage III is characterized by a distortion of the hepatic architecture with numerous fibrous septa, mainly portal-portal, sometimes portal-central fibrosis (Bridging fibrosis). Stage IV is defined by cirrhosis with the existence of regenerative nodules.

\section{Liver elastography:}

Liver Stiffness Measurement (LSM) with transient elastography (Fibro scan) has been shown as a simple, reliable surrogate marker of liver fibrosis in various chronic liver diseases. Though the effectiveness of this method is well established in patients with chronic hepatitis $\mathrm{C}$, only preliminary data are, so far, available in PBC. Recently, Corpechot et al [8]investigated the potential usefulness of transient elastography in the noninvasive evaluation of liver fibrosis stage and disease progression in PBC. They showed that the diagnostic thresholds of LSM in discriminating fibrosis stages F1, F2, F3, and $\geq$ F4 were 7.1, 8.8, 10.7, and $16.9 \mathrm{kPa}$, respectively. In addition, over a 5-year period, they found that progression of LSM in PBC is predictive of poor outcome. According to these findings, Liver Elastography should be used as a first level elastography. M Probe should be used whenever possible; in patients with obesity and skin-to-capsule thickness $>4 \mathrm{~cm}$ the $\mathrm{XL}$ probe should be used. Only results obtained on fasting state can be correctly interpreted. Confound- 
ers that can increase liver stiffness independent of fibrosis and should be taken into account are: liver inflammation, infiltrative diseases, cholestasis and venous congestion; probably fat content and iron content also increase liver stiffness.

Interpretation (in the absence of confounders): values of $\mathrm{LSM}>9.6 \mathrm{kPa}$ are associated with a 5-fold increased risk of liver decompensation, liver transplantation or death, and could be the cut-off to distinguish patients into early or advanced disease.

\section{Imaging assessment:}

Abdominal images are not necessary to confirm the diagnosis of PBC in patients who test positive for AMA. Nonetheless, imaging assessment with ultrasound (US), computed tomography (CT), or Magnetic Resonance (MRI) should be useful for excluding biliary obstruction, but first of all, abdominal US examination is mandatory in all patients with biochemical evidence of cholestasis to disclose intrahepatic or extra hepatic bile duct dilatation or focal liver lesions. There are no specific features of PBC on US; in particular the biliary tree appears normal. In addition, US findings in advanced PBC resemble those seen in other forms of cirrhosis. Indeed, if the diagnosis is uncertain, magnetic resonance cholangiopancreatography(MRCP) or endoscopic retrograde cholangiopancreatography (ERCP), may be necessary for a differential diagnosis, and specially to exclude primary sclerosing cholangitis or other biliary tract diseases.

\section{Patients Follow-up}

- $\quad$ Liver tests and clinical evaluation every 3-6 months

- $\quad$ Thyroid status and serological profile (AMA, ANA, SMA) annually

- Fibros can annually

- Bone mineral densitometry every 1-3 years

- Vitamins A, D, E, K annually if bilirubin $>2 \mathrm{mg} / \mathrm{dl}$

- Upper endoscopy every 1-3 years in patients with known or suspected cirrhosis

- Ultrasound \pm AFP every 6 months in patients with known or suspected cirrhosis

\section{PBC Treatment}

Liver transplantation is the only option for patients with PBC with life-threatening end-stage liver disease and its complications or severe intractable symptoms. Medical treatments of PBC have included primary therapies aimed at the underlying disease processes and at preventing or delaying progression, therapies for symptoms such as fatigue and pruritus to improve quality of life, and therapies directed at complications, including osteoporosis, fat-soluble vitamin deficiency, and complications of portal hypertension (such as cites, edema, varicealbleeding, and hepatic encephalopathy). Generally speaking, therapeutic opportunities in PBCare offered by targeting the so-called 'upstream' immune response, 'midstream' biliary injury leading tocholestasis and 'downstream' fibrotic processes[9]. Comprehensive practice guidelines have been published, representing consensus of expert opinion for the management of $\mathrm{PBC}(2)$.

There are no specific treatments for complications related to portal hypertension in PBC that differ from other causes of endstage liver disease, and they will not be discussed here. Therapy may also be needed for associated syndromes, such as thyroid disease, arthritis, or Jorgen syndrome, and they will also not be discussed here.

Ursodeoxycholic acid (UDCA), the first-line recommended pharmacotherapy for patients with $\mathrm{PBC}$, is able to ameliorate serum biochemical abnormalities and to potentially delay progression of disease to death or need for transplantation. According to the European guidelines the optimum dose is $13-15 \mathrm{mg} / \mathrm{kg}$ per day, which can be given as a single oral daily dose or divided doses if tolerability is an issue. UDCA very safe, with minimal side effects when administered to patients at its recommended dose (weight gain of about $3 \mathrm{~kg}$ in the first 12 months, hair thinning, and, rarely, diarrhea and flatulence are reported). It seems to be also safe during pregnancy and breast-feeding(2).

Recently, Obeticholic acid (OCA) - a farnesoid $\mathrm{X}$ receptor agonist - has been licensed as treatment options for patients with $\mathrm{PBC}$ [10]. In particular, European guidelines recommend it in combination with UDCA for patients with an inadequate response to UDCA, or as immunotherapy in those intolerant to UDCA. Its administration is recommended at a dose of $5 \mathrm{mg}$ with adjustment to $10 \mathrm{mg}$ if applicable, according to tolerability at 6 months. Treatment with OCA is associated with a dose dependent exacerbation in pruritus, leading to treatment discontinuation in $4-10 \%$ of patients. OCA-treated patients may also exhibit (reversible) alterations in serum lipid levels; specifically, a decrease in HDL coupled with an increase in total and LDL cholesterol [2].

Novel therapeutic approaches targeting PBC are under evaluation in several phases II-III studies, including budesonide and fabric acids derivates with anti-inflammatory, anti-fibrotic and metabolic effects[9].

Biochemical non-response to UDCA (mainly defined by bilirubin and ALP levels at1 year) is a strong prognostic factor in PBC.

In order to define the optimal time point for liver transplantation, the Mayo risk score [11] was validated as a reliable prognostic index for patients with PBC. This index was derived from analyses of large datasets, and is calculated from easily obtained clinical information: age, serum bilirubin, albumin, prothrombin time, and presence of edema. The prognostic value of the Mayo risk score has been validated for patients treated with UDCA, which improves serum biochemical values in many patients.

Nonetheless, in the last few years, novel tools for monitoring PBC and PSC patients have recently emerged. Their use represents a step forward in risk stratification in order to allow for more personalized care and adapted management as well as for well-designed therapeutic trials.

Generally, Paris criteria [12] are considered to have the best predictability of transplant-free survival as validated in large studies, such as from the Globe PBC Study Group(http://www. globalpbc.com/globe) and the UK PBC Consortium(www.ukpbc.com), and their usage has been recommended by an International Guidelines. Additionally, these 2 groups have led to development of new continuous scoring systems incorporating both measures of treatment response and parameters of disease severity, showing comparable risk quantification $[13,14]$.

\section{Research Progresses on the Horizon}

Understanding the biology of PBC is necessary for treating patients with a targeted approach. PBC pathogenesis is based on the interaction of immune and biliary pathways, progress- 
ing to injury - driving an inter-dependent and chronic cycle of cholestasis and liver fibrosis [2]. Additionally, several groups all over the world (Research Cohort - UK-PBC, PBC Foundation, GLOBAL PBC Study Group, and so on) are working on understanding the genetics of PBC. Case-control studies and genomewide association studies in human cohorts aim to elucidate PBC mechanisms by finding genetic variants that predispose a patient to PBC. However, the assembly of human cohorts requires the recruitment of a large number of patients as well as the elaborate assimilation of recruitment centers for disease characterization and accurate documentation, even if with some limitations, which were overcome by international collaboration and large consortia as well as more detailed phenotypic characterization and follow-up. In particular, in genome-wide association studies the strongest genetic associations in $\mathrm{PBC}$ are HLA associations $[15,16]$. Most non-HLA associations in PBC overlap with other autoimmune diseases, with risk loci indicating altered immunoregulatory pathways, dysregulated mucosal immunity, or aberrant microbial handling [17].

\section{Conclusions and Future Perspectives}

In the last 10 years, recent advances in PBC have attempted to improve the accuracy of the disease's diagnosis and prognosis, as well as the treatment options. Promising novel therapies (including fibroblast growth factor 19 and apical sodium-dependent bile acid transporter inhibitors, immunomodulators, T cells and interleukins modulators, fibrates and rituximab) are under evaluation in PBC patients on the basis of understanding thoroughly the cellular and molecular mechanisms touched upon in all histological stages of PBC, from the early autoimmunemediated bile duct epithelial cell damage to the destructive and illness-persistent influences of intrahepatic cholestasis, and finally giving rise to hepatic fibrosis and hepatic cirrhosis progression. Results of ongoing clinical trials will likely further improve medical management of PBC and stride toward accurate treatment in the near future.

\section{References}

1. Beuers U, Gershwin ME, Gish RG, et al. Changing nomenclature for PBC: from 'cirrhosis' to 'cholangitis'. Gastroenterology. 2015; 149: $1627-1629$.

2. European Association for the Study of the Liver. EASL Clinical Practice Guidelines: The diagnosis and management of patients with primary biliary cholangitis. J Hepatol. 2017; 67: 145-172.

3. Lindor KD, Gershwin ME, Poupon R, et al. American Association for Study of Liver Diseases. Primary biliary cirrhosis. Hepatology. 2009; 50: 291-308.

4. Jepsen P, Gronbaek L, Vilstrup H. Worldwide incidence of autoimmune liver disease. Dig Dis. 2015; 33: 2-12.
5. Boonstra K, Kunst $A E$, Stadhouders $P H$, et al. Rising incidence and prevalence of primary biliary cirrhosis: a large populationbased study. Liver Int. 2014; 34: e31-e38.

6. Dahlqvist G, Gaouar F, Carrat F, et al. Large-scale characterization study of patients with antimitochondrial antibodies but nonestablished primary biliary cholangitis. Hepatology. 2017; 65: 152-163.

7. Cheung KS, Seto WS, Fung J, et al. Epidemiology and Natural History of Primary Biliary Cholangitis in the Chinese: A TerritoryBased Study in Hong Kong between 2000 and 2015. Clinical and Translational Gastroenterology. 2017; 8: e116.

8. Corpechot C, Carrat F, Poujol-Robert A, et al. Noninvasive elastography-based assessment of liver fibrosis progression and prognosis in primary biliary cirrhosis. Hepatology. 2012; 56: 198208.

9. Dyson JK, Hirschfield GM, Adams DH, et al. Novel therapeutic targets in primary biliary cirrhosis. Nat Rev Gastroenterol Hepatol. $2015 ; 12: 147-158$

10. Nevens F, Andreone P, Mazzella G, et al. A placebo-controlled trial of obeticholic acid in primary biliary cholangitis. N Engl J Med. 2016; 375: 631-643.

11. Dickson ER,Grambsch PM,Fleming TR, et al. Prognosis in primary biliary cirrhosis: model for decision making. Hepatology. 1989; 10: 1-7.

12. Corpechot C, Chazouilleres O, Poupon R. Early primary biliary cirrhosis: biochemical response to treatment and prediction of long-term outcome. J Hepatol. 2011; 55: 1361-1367.

13. Lammers WJ, Hirschfield GM, Corpechot C, et al. Development and validation of a scoring system to predict outcomes of patients with primary biliary cirrhosis receiving ursodeoxycholic acid therapy. Gastroenterology. 2015; 149:1804-1812.

14. Carbone M, Sharp SJ, Flack S, et al. The UKPBC risk scores: Derivation and validation of a scoring system for long-term prediction of end-stage liver disease in primary biliary cirrhosis. Hepatology. 2016; 63: 930-950.

15. H.J. Cordell, Y. Han, G.F. Mells, et al. International genome-wide meta-analysis identifies new primary biliary cirrhosis risk loci and targetable pathogenic pathways. Nat. Commun. 2015; 6: 8019.

16. G.F. Mells, J.A. Floyd, K.I. Morley, et al. Genome-wide association study identifies 12 new susceptibility loci for primary biliary cirrhosis. Nat. Genet. 2011; 43: 329-332.

17. Reichert MC, Hall RA, Krawczyk M, et al. Genetic determinants of cholangiopathies: Molecular and systems genetics. Biochim Biophys Acta. 2017. 